\title{
Optimizing text for an individual's visual system: The contribution of visual crowding to reading difficulties
}

\author{
Sung Jun Joo ${ }^{1,2, *}$, Alex L. White ${ }^{3}$, Douglas J. Strodtman ${ }^{1,2}$, \& Jason D. Yeatman ${ }^{1,2}$ \\ ${ }^{1}$ Institute for Learning \& Brain Sciences, ${ }^{2}$ Department of Speech and Hearing Sciences, and \\ ${ }^{3}$ Department of Psychology, University of Washington, Seattle, WA 98195
}

Running title: Text-spacing, crowding and dyslexia

${ }^{*}$ Correspondence should be addressed to Sung Jun Joo at Institute for Learning \& Brain Sciences, Portage Bay Building, Box 357988, University of Washington, Seattle, WA 98195, USA, E-mail: sjjoo@uw.edu 


\section{Abstract}

Reading is a complex process that involves low-level visual processing, phonological processing, and higher-level semantic processing. Given that skilled reading requires integrating information among these different systems, it is likely that reading difficulty—known as dyslexia—can emerge from impairments at any stage of the reading circuitry. To understand contributing factors to reading difficulties within individuals, it is necessary to diagnose the function of each component of the reading circuitry. Here, we investigated whether adults with dyslexia who have impairments in visual processing respond to a visual manipulation specifically targeting their impairment. We collected psychophysical measures of visual crowding and tested how each individual's reading performance was affected by increased textspacing, a manipulation designed to alleviate severe crowding. Critically, we found that many individuals with dyslexia showed elevated crowding, and that these individuals read faster when text was rendered with increased letter-, word- and line-spacing. Our findings point to a subtype of dyslexia involving elevated crowding and demonstrate that individuals with dyslexia benefit from interventions personalized to their specific impairments.

Keywords: dyslexia, visual crowding, visual attention, text spacing, reading 


\section{Introduction}

Reading involves multiple stages of processing, including low-level sensory processing of the visual stimulus, phonological processing of the sounds associated with the printed letters, and higher-level semantic processing of the meaning of words and sentences. The complex and multi-faceted nature of reading suggests that impairments at any processing stage could cause difficulties with reading (Joo, Donnelly, \& Yeatman, 2017; Paulesu et al., 2001; Pennington, 2006; Pennington \& Bishop, 2009; Peterson \& Pennington, 2012; Siok, Perfetti, Jin, \& Tan, 2004).

However, theories of dyslexia have been attempting to uncover a single underlying deficit. This effort has been frustrated by discrepant results obtained from similar experimental paradigms. For example, whether dyslexic readers have poor motion sensitivity or elevated visual crowding compared to typical readers has been a subject of heated debate (Amitay, BenYehudah, Banai, \& Ahissar, 2002; Bouma \& Legein, 1977; Demb, Boynton, \& Heeger, 1997; Doron, Manassi, Herzog, \& Ahissar, 2015; Eden et al., 1996; Joo et al., 2017; Lovegrove, Bowling, Badcock, \& Blackwood, 1980; Martelli, Di Filippo, Spinelli, \& Zoccolotti, 2009; Olulade, Napoliello, \& Eden, 2013; Skottun, 2000; Stein \& Walsh, 1997). Furthermore, the phonological impairment theory of dyslexia cannot explain the collection of visual deficits reported in people with dyslexia unless these deficits are epiphenomenal and not causally related to reading skills (Franceschini, Gori, Ruffino, Pedrolli, \& Facoetti, 2012; Ramus et al., 2003; Vellutino, Fletcher, Snowling, \& Scanlon, 2004; Vidyasagar \& Pammer, 2010). Given the complex nature of reading, the lack of replication across previous studies could be indicative of heterogeneity in dyslexia (Pennington, 2006)_individuals struggle with reading for a variety of reasons_instead of supporting or rejecting any specific theory.

Individual differences in dyslexia suggest that it is essential to characterize the function of multiple components of the reading circuitry in order to understand the factors contributing to 
an individual's reading difficulties (Wandell \& Le, 2017). In the present study, we sought to diagnose limitations in an individual's visual system, an input system in reading circuitry, and establish the link between deficits in visual processing and reading difficulties. Beyond demonstrating a correlation between visual processing and reading skills, the critical test should show that individuals with a specific impairment respond to an intervention designed to ameliorate that impairment.

We first assessed limitations in the individual's visual system using a visual crowding paradigm in which the ability to identify an object is deteriorated by nearby items (Whitney \& Levi, 2011), and then assessed the relation between individual differences in reading ability and visual crowding. We then tested whether increased text-spacing, which provides a less crowded visual environment, improves reading specifically for those who have elevated crowding. There is an appealing link between crowding and reading because successful decoding of single words in a crowded page of text is essential for skilled reading. In a typical page of text, visual crowding is always present because letters outside the fovea crowd each other. Indeed, crowding is a determining factor for the number of letters that can be recognized in a single fixation for typical readers (Legge, Mansfield, \& Chung, 2001; Pelli \& Tillman, 2008; Yu, Cheung, Legge, \& Chung, 2007) and is negatively correlated with reading skills in children with dyslexia (Bouma \& Legein, 1977; Gori \& Facoetti, 2015; Martelli et al., 2009; Spinelli, De Luca, Judica, \& Zoccolotti, 2002). Furthermore, increased text-spacing, on average, helps dyslexic children perform a lexical decision task and read sentences out loud (Perea \& Gomez, 2012a; Zorzi et al., 2012). However, it is not clear whether this text-spacing effect is related to general low reading skills, or specific to a subset of individuals with elevated crowding.

We identified individuals who have both elevated crowding and reading difficulties, and found that increased text-spacing results in better reading performance specifically for those individuals. As a control analysis, we used a cued visual search paradigm to rule out impaired spatial attention as the mechanism underlying our results. These findings point to a subtype of 
dyslexia potentially caused (or exacerbated) by elevated crowding and suggests that personalizing the reading environment by adjusting the properties of the visual input (e.g., increased text-spacing) at the front end of reading process can improve reading performance.

\section{Methods}

All procedures, including recruitment, consent, and testing, followed the guidelines of the University of Washington Human Subjects Division and were reviewed and approved by the UW Institutional Review Board. To ensure reproducibility of our results, all experimental procedures, data, and analysis code are available in the study's github repository (https://github.com/YeatmanLab/ProvidedUponPublication).

\section{Subjects}

To recruit a sample of adults with heterogeneous reading abilities we posted flyers soliciting research participants with and without dyslexia. Flyers were also disseminated through local organizations that provide support to people with dyslexia including the University of Washington Disability Resources for Students (DRS), Disabilities, Opportunities Internetworking and Technology (DO-IT), and Dyslexic Advantage (http://www.dyslexicadvantage.org/). Since there is no agreed upon definition of dyslexia, we used quantitative measures of reading abilities for all of our analyses rather than relying on specific criteria for dyslexia. We conducted a power analysis to calculate a priori sample size required to result in the power of 0.8 (with type $\mathrm{I}$ error $=$ 0.05 and Type II error $=0.20)$ for our main data analysis (independent t-test between groups). This analysis yielded 34 subjects (17 in each group). A total number of 39 adults (aged $30 \pm 11 \mathrm{y}$, 23 females, 15 males, and 1 unspecified) participated in the crowding and reading experiments (29 reported having dyslexia or reading difficulties). This sample size is large enough to robustly detect the group differences of our manipulation. Thirty seven adults from this subject pool took part in the cueing experiment. Thirty eight children (aged 9.6 $\pm 1.8 \mathrm{y}, 16$ females and 22 males) 
participated in the crowding experiment. All subjects had normal or corrected-to-normal vision. and gave informed written consent in accordance with the University of Washington Institutional Review Board. A spreadsheet containing all behavioral measurements and subject demographics is available as a .csv file within the study's github repository.

\section{Apparatus}

Stimuli were created using MATLAB (The Mathworks Corporation, Natick, MA, USA) in conjunction with the Psychophysics Toolbox (Brainard, 1997; Pelli, 1997) on a Linux PC (Mint Mate, version 17). Stimuli were displayed on a LG liquid crystal display $(1920 \times 1080$ resolution, $120 \mathrm{~Hz}$ refresh rate, subtending $50^{\circ}$ horizontally at viewing distance of $58 \mathrm{~cm}$ ). The subjects' response was collected using a joystick in the crowding experiment and a computer keyboard in the cueing experiment.

\section{Stimuli and Procedure}

Crowding experiment. Stimuli comprised four open circles (flankers; $1^{\circ}$ diameter, $0.08^{\circ}$ linewidth) and an open circle with a gap (target; an arc with reflex central angle of $330^{\circ}$ ). All Stimuli were black $\left(0 \mathrm{~cd} / \mathrm{m}^{2}\right)$ and displayed on a gray background $\left(135 \mathrm{~cd} / \mathrm{m}^{2}\right)$. The target eccentricity was defined by the center-to-center distance between the fixation mark at the center of display and the target. There were two target eccentricity conditions (near: $6^{\circ}$, far: $10^{\circ}$ ). We measured crowding effects at each target eccentricity separately. To quantify crowding effects, we defined critical spacing as the minimal center-to-center distance (threshold) between a target and flankers at which the observer can report the target identity at $82 \%$ correct. We used an adaptive staircase (QUEST; (Watson \& Pelli, 1983)) to estimate individual's critical spacing. In an experimental session, a target eccentricity was fixed and the initial starting target-flanker distance was set as 1.3 times greater than half the eccentricity $\left(3.9^{\circ}\right.$ for $6^{\circ}$ eccentricity; $6.5^{\circ}$ for $10^{\circ}$ eccentricity). The subsequent target-flanker distance was controlled by the QUEST 
procedure. There were two independent staircases (25 trials each) to prevent subjects from predicting the difficulty of the next trial. Each subject finished 4-6 session for each target eccentricity. The order of target eccentricity in each subject was alternated across sessions, and we counterbalanced the session order of which target eccentricity a subject encountered first across subjects. On a given trial, the fixation mark was displayed first and remained in the display for the entire trial. After 500 ms of fixation onset, the stimuli were displayed either the left or the right side of display for $150 \mathrm{~ms}$. After the stimulus offset, only the fixation mark was displayed until subjects made a response: subjects reported the direction of the gap (either upward or downward) in the target by moving a joystick up (upward) or down (downward). Visual and auditory feedback was given for both correct (the fixation mark changed to '+' sign with a designated tone for correct responses) and incorrect (the fixation mark changed to '-' sign with a designated tone for incorrect responses). There was a $1 \mathrm{~s}$ blank between feedback and the beginning of the next trial.

Cueing experiment. The search array consisted of eight Gabor patches: $50 \%$ contrast grayscale sinusoidal gratings with spatial frequency 2 cycles $/^{\circ}$, windowed by a $2 \mathrm{D}$ gaussian with standard deviation $0.28^{\circ}$. The Gabors were equally spaced around an imaginary circle, centered on the fixation mark with $5 \mathrm{deg}$ radius. The fixaiton mark was a black plus symbol $0.3^{\circ}$ wide. On each trial, 7 of the Gabors were oriented vertically, and one (the target) was tilted. The position of the target was chosen randomly on each trial. The observer's task was to report the direction of the target's tilt (clockwise or counterclockwise from vertical) by pressing one of two keys. The magnitude of the tilt was controlled by a weighted up/down staircase that converged on a $75 \%$ correct threshold (García-Pérez, 1998).

Each trial began with a cue interval for $33 \mathrm{~ms}$. In the uncued condition, this interval contained only the fixation mark. In the cued condition, it also contained a dark red dot $0.6^{\circ}$ in diameter, at $3^{\circ}$ eccentricity along the imaginary line that connected the fixation mark to the upcoming target. The cue interval was followed immediately by the search array for $83 \mathrm{ms,} \mathrm{and}$ 
then an open-ended response interval that ended when the observer pressed one of the two keys. A 75 ms tone provided immediate feedback (high vs. low pitch for correct vs. incorrect). In addition, the observer gained 3 points for each correct response and 0 points for each incorrect response. The number of points gained was displayed for $750 \mathrm{~ms}$ in green or red text immediately after the keypress. After an intertrial interval of $580 \mathrm{~ms}$, the next trial began. The cued and uncued conditions were separated into blocks of 52 trials. At the end of each trial the total number of points gained was printed on the screen. Each observer completed two blocks of each condition.

Reading experiment. Real words were selected from the MRC Psycholinguistic Database (http://websites.psychology.uwa.edu.au/school/MRCDatabase/uwa_mrc.htm) by specifying the length in letters (between four and eight letters) and the Kucera-Francis frequency $(>125)$. Words were selected by an author (DJS) with the goal of choosing words that were easily recognized, culturally neutral, and representative of a wide range of word forms. Longer lists of words were reviewed by 3 independent native English speakers (graduate students and postdocs who studied linguistics) and words indicated as being unfamiliar or more difficult were removed. Thirty two words of each length were selected for the final lists. Pseudowords were generated using MCWord Database (http://www.neuro.mcw.edu/mcword/) by specifying the length in letters (between four and eight letters) and generating constrained trigram-based strings. The lists of words were reviewed by the same 3 independent native English speakers and words indicated as being more difficult or having unclear pronunciations were removed. Thirty two words of each length were selected for the final lists.

We created four real word and pseudoword lists containing 40 words with normal and increased text-spacing (a total of 16 lists). We used Calibri font (11pt) for normal spacing and Fluent Calibri font (11pt; https://www.microsoft.com/en-us/download/details.aspx?id=50721) for increased spacing. Compared to 11 point Calibri, 11 point Fluent Calibri increases the letter 
space by 1.375 point, triples the size of the word space, and a complete 11 point empty line between each line of text.

Subjects completed this experiment across two sessions separated by at least a week. In each session, subjects were asked to read the 8 lists (4 real word and 4 pseudoword lists). In one session odd numbered lists were with normal text-spacing and even numbered lists were with increased text-spacing, and in the subsequent session the text-spacing condition was flipped. This order was counterbalanced across subjects. Subjects were instructed to read the words on the list as fast and accurate as possible.

We measured the start time when the subject began saying the first word on the list and the stop time when they completed the last word. We used strict criteria for accuracy for real words (taking into account dialectal and articulatory differences). We marked correctness of each word during the session. On pseudowords, we used more liberal criteria. The goal was to make sure that individuals were decoding letters in the right order and applying rules of English phonology. Between each list we made sure to check in with the subject to ensure that they were ready to continue, providing them as much time as they needed before beginning the next list. PDFs of the word exact stimuli used in this experiment can be found in the study github repository.

\section{Standardized reading assessment}

We administered a battery of behavioral tests including subtests from the Wechsler Abbreviated Scales of Intelligence, Comprehensive Test of Phonological Processing, Test of Word Reading Efficiency and the Woodcock Johnson IV Tests of Achievement. Here we used the Test of Word Reading Efficiency (TOWRE) composite score because it includes measures of real word and pseudoword reading in a timed manner. Most of adult subjects were highly compensated university students and Woodcock Johnson scores were not suitable to distinguish reading ability among highly compensated individuals with dyslexia. 
Data analysis

For the crowding experiment, we used QUEST (Watson \& Pelli, 1983) staircases to generate the center-to-center distance for each trial. We re-fitted the psychometric function using QUEST procedure by setting the threshold and the slope as free parameters. We excluded staircases in which threshold estimate was greater than the maximum flanker-target distance $\left(6^{\circ}\right.$ or $\left.10^{\circ}\right)$ suggesting the staircase is not converging. We also excluded staircases with a threshold estimate being less than the minimum flanker-target distance $\left(1^{\circ}\right)$ where the flankers and the target overlap. Applying these criteria discarded a total number of 69 staircases out of 400 staircases, resulting in 4-6 staircases per eccentricity for each subject. We averaged the threshold estimates of those remaining staircases to define each subject's critical spacing. Including staircases with threshold estimate $<1^{\circ}$ did not change the results.

For the cueing experiment, we estimated $75 \%$ correct thresholds with maximum likelihood fits of Weibull psychometric functions to the raw data. To quantify the attention effect, we defined attention index as follows: Attention index $=1-$ Thresh $_{\text {cued }} /$ Thresh $_{\text {uncued. }}$ The higher the attention index, the greater the attention effect.

For the reading experiment, we discarded the pseudoword reading time greater than 3 SD above the mean. This procedure removed one subject as an outlier. All the analyses were based on the remaining 38 subjects.

\section{Results}

\section{Elevated crowding predicts reading difficulties}

We first sought to address the relationship between visual crowding and reading skills. Crowding has been suggested as a potential cause of dyslexia based on data showing that dyslexic subjects experience, on average, elevated crowding compared to typical readers (Bouma \& Legein, 1977; Martelli et al., 2009). However, previous studies used letters to 
measure crowding, so differences in crowding might represent linguistic deficits in dyslexic subjects. To remove this potential confound, we measured crowding using non-linguistic stimuli (Figure 1A; see Method for details). The stimuli comprised circles (flankers) and a circle with a gap (target). On each trial, subjects report which direction the gap in the target was facing (up or down). The magnitude of crowding that an individual experiences was indexed by critical spacing, which is the minimal distance (threshold) between the target and flankers at which the observer can report the target identity.

Critical spacing increased as the eccentricity of the target increased (Figure 1B), confirming conventional eccentricity-dependent crowding effects (Bouma, 1970; Levi, 2008; Whitney \& Levi, 2011). With this simple perceptual judgment, critical spacing at near eccentricity $\left(6^{\circ}\right)$ was negatively correlated with reading skills (Figure $1 \mathrm{C} ; r=-0.37, p=0.02$ ): subjects with high critical spacing (more crowding) have worse reading skills than subjects with low critical spacing (less crowding). Critical spacing at far eccentricity $\left(10^{\circ}\right)$ was not correlated with reading skills (Figure 1D; $r=-0.07, p=0.67$ ). To ensure that these findings do not reflect specific characteristics of our adult sample, we ran a replication experiment in an independent sample of children $(\mathrm{N}=38,9.6 \pm 1.8 \mathrm{y})$, and confirmed that critical spacing at near eccentricity is correlated with reading skills $(r=-0.42, p=0.01)$. We focused on critical spacing at near eccentricity for subsequent analyses because crowding in the far periphery is less relevant for reading. 
A
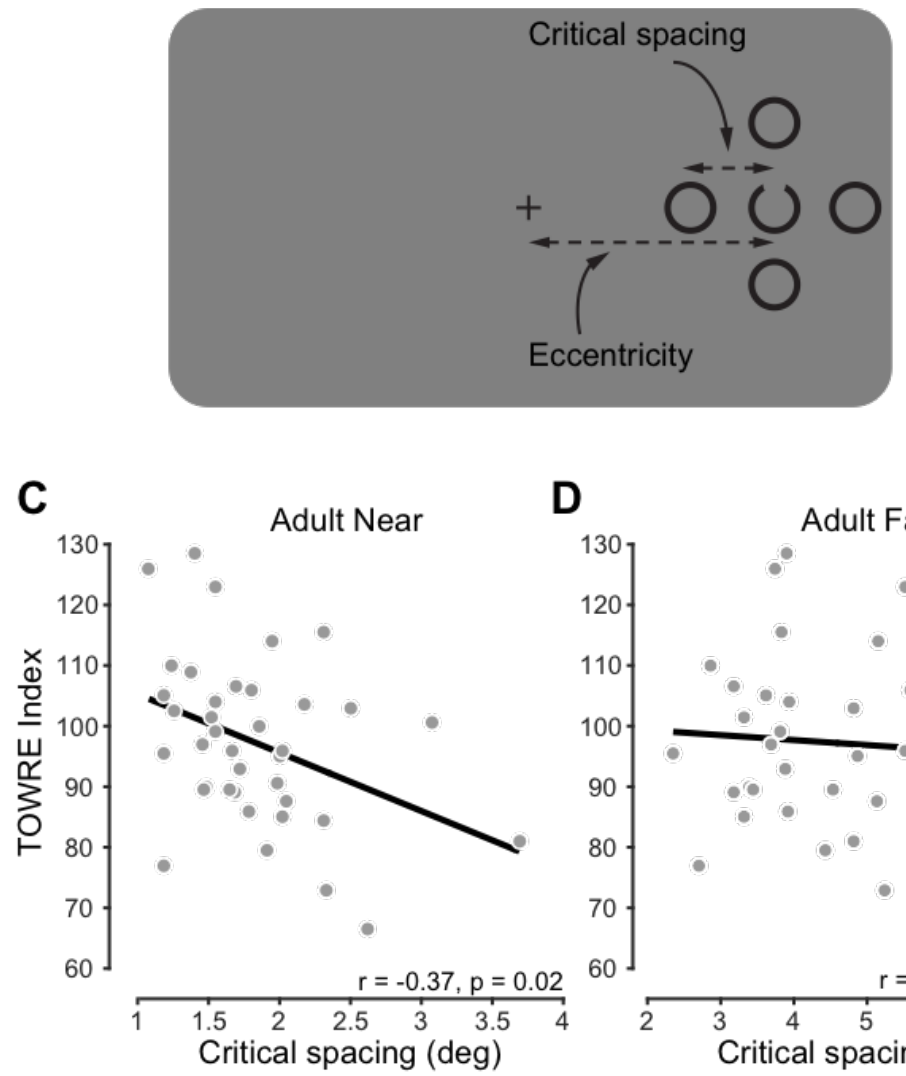
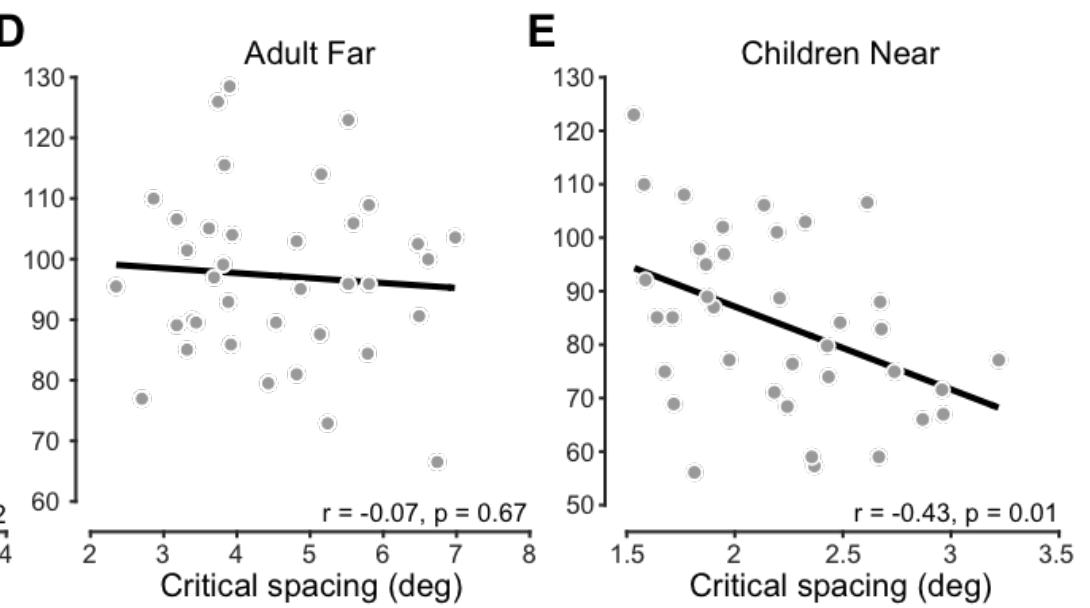

Figure 1. Crowding is correlated with reading skills. (A) Crowding stimuli. After $500 \mathrm{~ms}$ of fixation on the central mark, crowding stimuli appeared at either the left or the right side of the display. The stimuli disappeared after $150 \mathrm{~ms}$ and subjects reported the direction of the gap (up or down) using a joystick. We measured critical spacing at two target eccentricities $\left(6^{\circ}\right.$ or $\left.10^{\circ}\right)$ in separate blocks of trials. (B) Critical spacing increases with target eccentricity (means: 1.82 at $6^{\circ} ; 4.55$ at $10^{\circ}$ ). The error bars represent bootstrapped $68 \%$ confidence intervals (Cls). (C-D) The relationship between reading skills and critical spacing in adults. The $y$-axis indicates the TOWRE composite score, a standardized index of reading speed and accuracy that is normed by age (population mean $=100, S D=15$ ). The $x$-axis represents critical spacing at $6^{\circ}(C)$ and $10^{\circ}(D)$ eccentricity. Gray circles show individual data points, and the black line is the best-fitting linear regression line to the data. (E) The relationship between reading skills and critical spacing in children. The $x$-axis indicates critical spacing at $6^{\circ}$ eccentricity.

\section{Increased text-spacing improves reading performance for adults with elevated crowding}

The data presented above confirm previous reports that crowding and reading ability are correlated (Bouma \& Legein, 1977; Martelli et al., 2009), and our data further rule out alternative explanations of this phenomenon by using non-linguistic stimuli. But is this relationship causal? To determine if crowding contributes to individual differences in reading abilities, we experimentally manipulated spacing between letters, words, and lines of text (Figure 2A-D, see 
Method for details). Even though inter-letter spacing does not affect the number of letters that fit into an individual's uncrowded window (Pelli \& Tillman, 2008), we hypothesized that increased letter, word and line spacing would alleviate the detrimental effects of a crowded visual environment for an impaired visual system (see Discussion for further details). Based on our hypothesis, we predicted that the extent to which an individual benefits from increased textspacing would be dependent on their critical spacing.

A

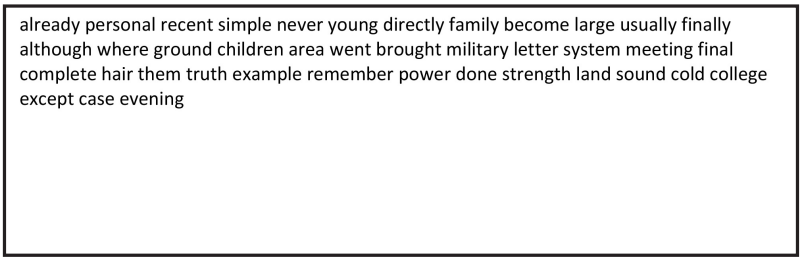

C

ordular childrow empand cormly thesh greep valaying insing strust cleps greetch glaying identant banth crents verating dard nump bastily vackling befort sigger sibbing flace crinking lant felp blans gettled probling theep noth preaming whem walps dond plassed pasked pess foldery

\section{B}

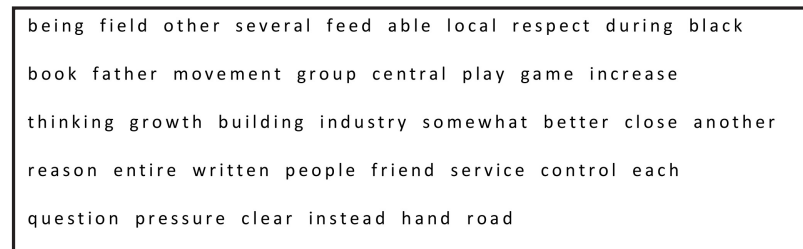

D

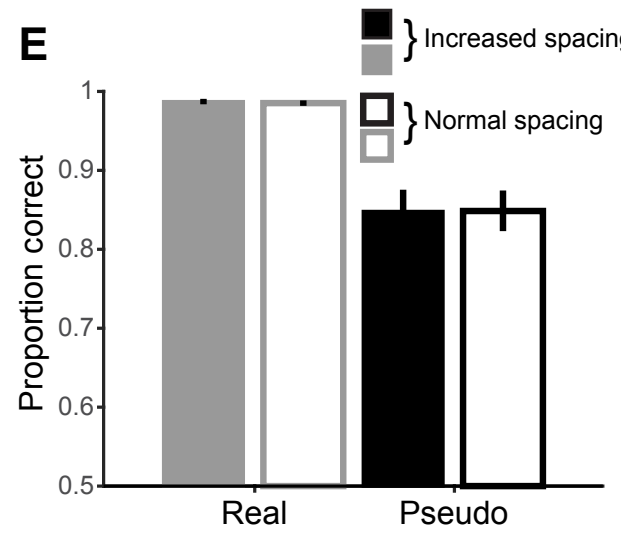

$\mathbf{F}$

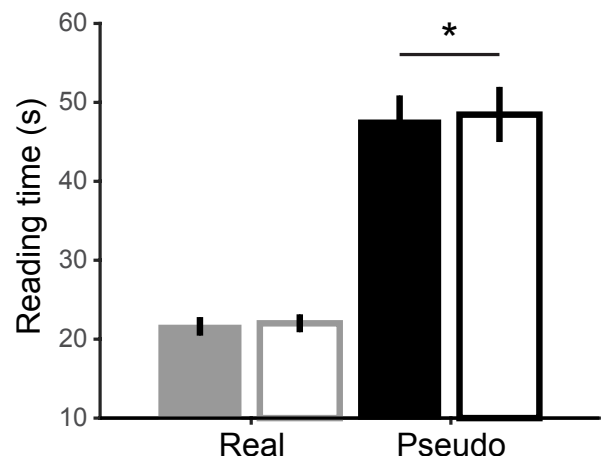

Figure 2. Increased text-spacing improves reading performance. $(A-D)$ Examples of real word $(A, B)$ and pseudoword $(C, D)$ lists with normal $(A, C)$ and increased $(B, D)$ text-spacing. (E) Accuracy for each list. Gray and black bars represent proportion correct for real words and pseudowords, respectively. Filled and empty bars indicate increased and normal text-spacing, respectively. Accuracy was not affected by text-spacing. Error bars are bootstrapped $68 \%$ Cis, representing the between subject variance (equivalent to \pm 1 SEM). (F) Reading time for each list. Increased text-spacing lowered reading time for pseudowords within individuals. Error bars are bootstrapped $68 \% \mathrm{Cls} .{ }^{*} p<0.05$ based on a paired sample t-test. 
Consistent with well-known word-frequency effects, there was a large difference in reading speed and accuracy for real versus pseudowords (pseudowords can be thought of as zero-frequency words). Accuracy was higher for high-frequency real words compared to pseudowords (Figure 2E; 98.6 $\pm 0.3 \%$ versus $84.8 \pm 2.7 \% ; F(1,37)=31.74, p=2 \times 10^{-6}$ ). Reading speed was faster for real words compared to pseudowords (Figure 2F; 22.14 \pm 1.17 ms versus $\left.50.34 \pm 3.98 \mathrm{~ms} ; \mathrm{F}(1,37)=83.96, \mathrm{p}<10^{-7}\right)$. Critically, increased text-spacing decreased reading speed for pseudowords $(t(37)=2.23, p=0.03)$ but not for real words $(t(37)=1.58, p=0.12)$. Accuracy did not depend on text-spacing for real words or for pseudowords (no main effect of text-spacing; $F(1,37)=0.18, p=0.67)$ ), indicating that text-spacing effects on reading speed were not due to a speed-accuracy trade-off.

We next examined whether the effect of text-spacing on pseudoword reading speed depended on reading ability. Only the subjects with low reading skills (TOWRE score < 95) benefitted from increased text-spacing (Figure 3A). The mean pseudoword text-spacing effect (difference in reading times for increased - normal spacing) for dyslexic readers was $1.91 \mathrm{~s}$ versus $0.11 \mathrm{~s}$ for typical readers $(t(36)=2.55, \mathrm{p}=0.02)$. Increased text-spacing did not affect reading speed for high-frequency real words in either group (Text-spacing effects for dyslexic versus typical readers: $0.54 \mathrm{~s}$ versus $0.27 \mathrm{~s} ; \mathrm{t}(36)=0.53, \mathrm{p}=0.59$ ). 

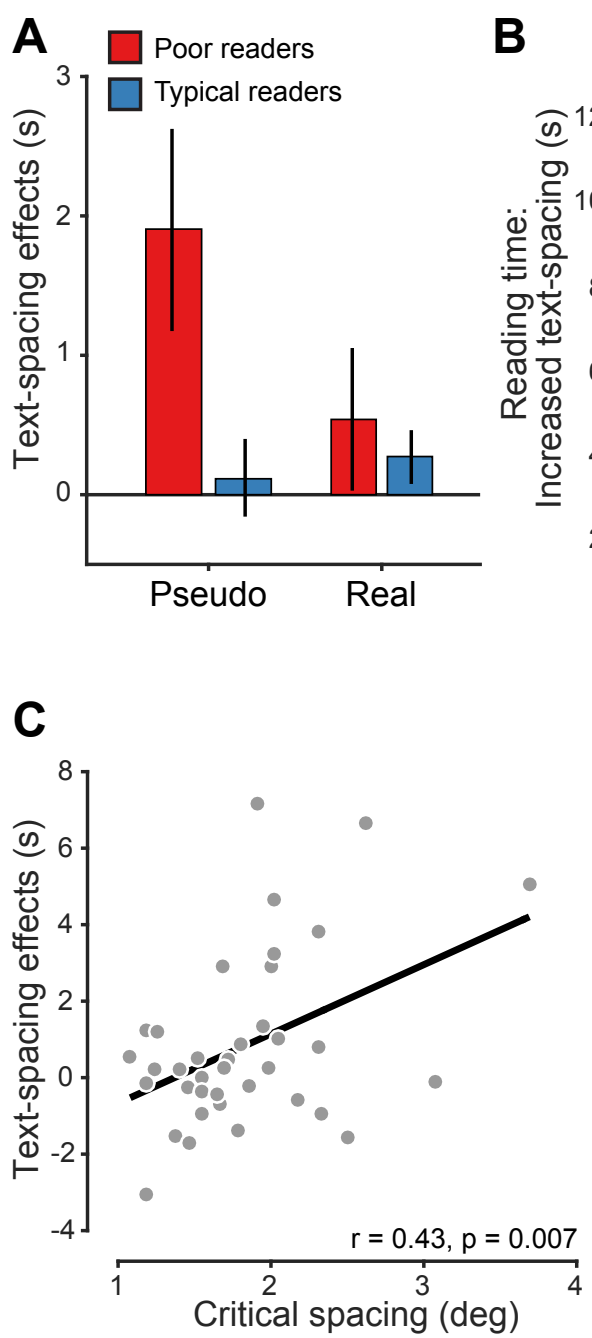

B

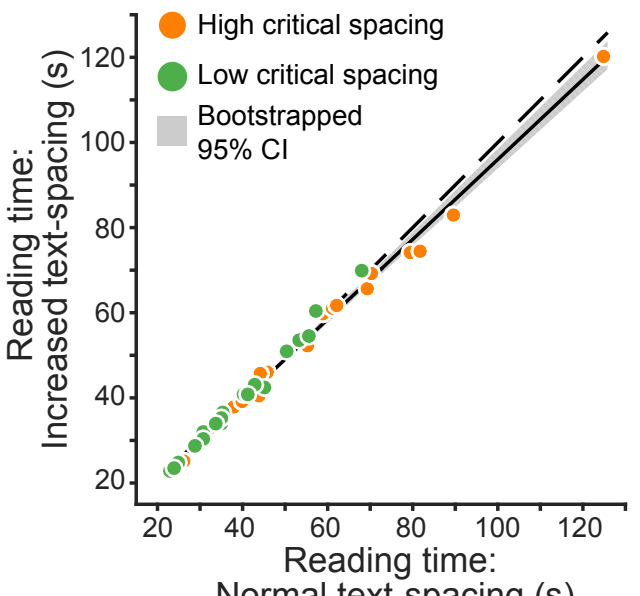

Normal text-spacing (s)

D

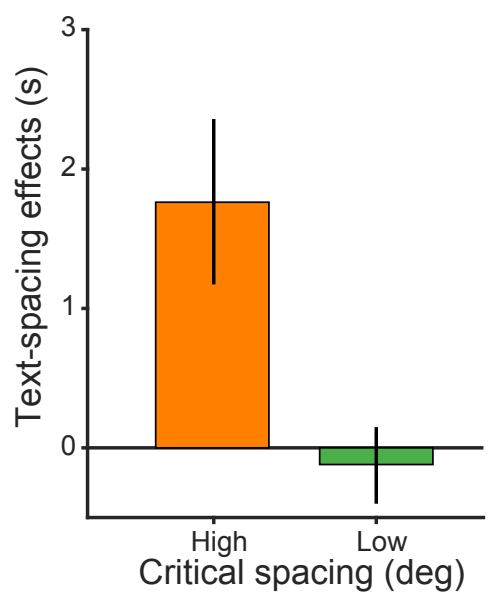

Figure 3. Increased text-spacing improves reading speed for poor readers with elevated crowding. (A) Subjects with low reading skills, but not typical readers, benefit from increased text-spacing. The $y$-axis is the difference between reading time for normal and increased text-spacing. Red and blue bars are for poor readers and typical readers, respectively. Poor readers: TOWRE index standard score < 95; Typical readers: TOWRE > 95. (B) Reading time for pseudowords with normal (x-axis) and increased (y-axis) text-spacing. Orange points represent individuals with high critical spacing and green points represent individuals with low critical spacing, based on a median split. The shaded region is a bootstrapped 95\% confidence interval for the best-fitting line. The dashed line is the unity line. For slow readers there is a substantial benefit of increased text -spacing, demonstrated by the significant deviation of the linear fit from the identity line. (C) Critical spacing predicts the effect of text-spacing. The black line is the bestfitting regression. (D) Text-spacing benefits are pronounced for subjects with high critical spacing and not present in the low critical spacing group. Error bars are bootstrapped $68 \% \mathrm{Cls}$.

Next, we assessed whether a subject's critical spacing is the main predictor of their textspacing effect. Figure 3B depicts individual reading times for pseudoword lists with increased and normal text-spacing. Critically, individuals with high critical spacing (orange) show textspacing effects—data points are below the unity line-while individuals with low critical spacing 
(green) do not show improvement—data points are above or on the unity line. Consistent with this observation, critical spacing was correlated with text-spacing effects (Figure $3 C ; r=0.43, p$ $=0.007$ ) suggesting that only subjects with elevated crowding benefit from increased textspacing. Does the correlation between critical spacing and text-spacing effects simply reflect a relationship between text-spacing effects and reading skills, given that critical spacing is also correlated with reading skills (Fig. 1C)? To rule out this possibility, we conducted a multivariate linear regression analysis using critical spacing and reading skills as independent predictor variables of the text-spacing effect. The results revealed that critical spacing is an independent predictor of text-spacing effects $(p=0.048)$ and reading skills are marginally significant $(p=$ 0.061). In other words, critical spacing predicts the text-spacing benefit even after controlling for differences in reading skills. We confirmed this finding with a stepwise regression analysis using a bidirectional elimination procedure (Draper \& Smith, 1998), which indicated that critical spacing is the main predictor of text-spacing effects. More importantly, text-spacing benefits were greater for subjects with high critical spacing compared to subjects with low critical spacing (Figure 3D; $1.76 \mathrm{~s}$ vs. $-0.12 \mathrm{~s}$ for high vs. low critical spacing, $t(36)=2.78, p=0.008$, cohen's $d$ $=0.9$ ), demonstrating that elevated crowding, regardless of reading skills, is a significant predictor of text-spacing effects.

\section{Impaired visual attention does not predict text-spacing effects}

We have demonstrated that poor readers with elevated crowding show better reading performance with increased text-spacing, suggesting that increased text-spacing alleviates crowding for these individuals. However, an alternative mechanism that could explain our results involves spatial attention (Grainger, Dufau, \& Ziegler, 2016; Vidyasagar \& Pammer, 2010). Several studies have shown that dyslexic readers have impairments in visuospatial attention (Bosse, Tainturier, \& Valdois, 2007; Franceschini et al., 2012; Roach \& Hogben, 2004). It is possible that visuospatial attention is necessary to select one word from neighboring text 
during reading. Those with an attentional deficit would therefore benefit from increased textspacing. Under this hypothesis, if increased crowding is due to an attentional deficit, some theories would interpret it as an indication of a general "dorsal stream dysfunction" (Vidyasagar \& Pammer, 2010).
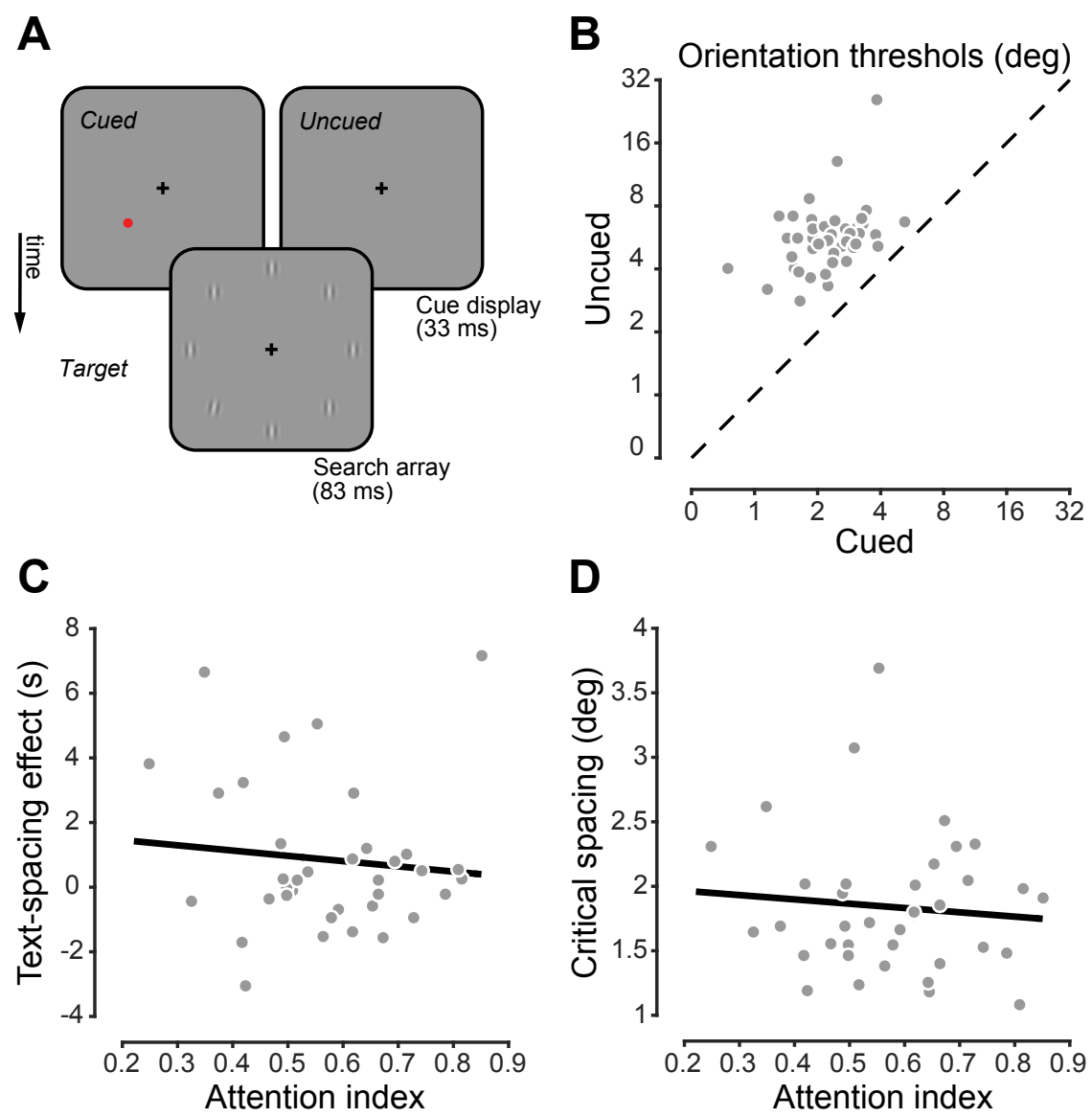

Figure 4. Text-spacing effects are not due to impaired spatial attention. (A) The stimuli and procedure for the cueing experiment. The search array (83 $\mathrm{ms}$ ) comprised 7 vertically oriented distractor Gabors and a tilted target Gabor. Observers reported the direction of the target's tilt. In the cued condition, a $100 \%$ valid peripheral pre-cue (red dot) appeared in the 33 ms preceding the search array. In the uncued condition there was no pre-cue. Orientation thresholds were measured with a staircase procedure (See Methods). (B) Orientation thresholds in the cued and uncued conditions. The dashed line represents the unity line. All subjects' thresholds were lower in the cued condition, suggesting that our subjects effectively used the cue to focus spatial attention and exclude distractors. (C) The attention index (cueing effect) does not predict text-spacing effects. (D) The attention index does not predict critical spacing.

To test this possibility, we used a cued visual search paradigm (Roach \& Hogben, 2004) in which subjects judge the orientation of a tilted Gabor patch in an array of seven vertical distractors (Figure 4A). All subjects had lower orientation thresholds in the cued than uncued 
condition, indicating that the cue was effective to direct attention to the target location (Figure 4B). However, there is no relationship between the attention index and either text-spacing effects (Figure 4C; $r=-0.10, p=0.54$ ) or critical spacing (Figure 4D; $r=-0.09, p=0.60$ ). Thus, we conclude that the text-spacing manipulation does not target attentional deficiencies in dyslexia.

\section{Discussion}

Our results show that crowding correlates with reading skills in adults and children, and that adult dyslexic readers with elevated crowding benefit from an experimental manipulation that alleviates crowding in printed text. The reading improvement conferred by text-spacing is not explained by an impairment in selective attention measured using a cueing paradigm. More generally, crowding and selective attention do not reflect a common mechanism. Thus, our results indicate that visual crowding is one mechanism that contributes to reading difficulties in a subset of people with dyslexia. It is important to note that the reverse inference-dyslexic readers have elevated crowding-does not logically follow from our conclusion. In fact, many children and adults with poor reading skills have low critical spacing (less crowding) (Figure 1C). This is consistent with previous data showing that only a subset of people with dyslexia have elevated crowding while a majority of people with dyslexia show crowding effects within the typical range (Bouma \& Legein, 1977; Doron et al., 2015; Martelli et al., 2009). While previous studies have argued that the overlapping distribution of critical spacing values between dyslexic and control subjects indicates that crowding does not contribute to dyslexia, our data offer a different interpretation. Rather than interpreting an individual's critical spacing as evidence for or against a deficit that characterizes dyslexics as a group, we demonstrate that critical spacing is indicative of limits on information processing within that individual's visual system. Our results suggest that there are some individuals with reading difficulty due to the characteristics of their 
visual system and that a simple manipulation in visual input (i.e., text-spacing) improves their reading behavior.

One of the influential theories of crowding suggests that increased spacing between letters will not help alleviate crowding because increasing spacing puts letters further into the periphery where crowding is greater, effectively canceling out the letter-spacing benefit (Herzog, Sayim, Manassi, \& Chicherov, 2016; Pelli \& Tillman, 2008). Consistent with this theoretical standpoint, typical readers in our study did not show any improvements in reading with increased text-spacing, and poor readers did not show any improvements when they read highfrequency real words (Figure 3A). So what made poor readers benefit from increased textspacing for pseudowords? First, in an impaired visual system there may not be the characteristic linear relationship between critical spacing and eccentricity (Pelli \& Tillman, 2008; Whitney \& Levi, 2011). Subtle nonlinearities would affect the optimal text-spacing. Second, and consistent with Pelli and colleagues (Pelli \& Tillman, 2008), it is not likely that more letters are being processed in a single fixation with increased text-spacing. Rather, increased text-spacing may reduce the crowding from the neighboring letters at the boundary of the uncrowded window, making it easier to select a group of letters from within a word. Reading pseudowords requires phonological decoding of letters into sounds, and for dyslexic readers, phonological decoding is an effortful process of chunking a word into pronounceable groups of letters. In an impaired visual system, with elevated crowding, nearby letters and words are likely to interfere with the process of selectively processing chunks of letters during phonological decoding. These interpretations remain open questions, and future research will shed light on how the subtle differences in the visual systems of people with dyslexia play a role in processing letters, chunks of letters, and the whole word in text with increased spacing.

How are our results related to natural reading? Normal text comprises words with frequency ranging from low to high, and pseudowords can be viewed as the extreme case of low frequency words, or words that a novice reader does not yet know. Our results suggest that 
people with elevated crowding would benefit from increased text-spacing while learning to read or when reading sentences that contain low frequency words (Perea \& Gomez, 2012b). Furthermore, increasing text-spacing not only facilitates natural reading speed but also decreases reaction time in lexical decision experiments (Perea \& Gomez, 2012a). Finally, a crowded page of text in the periphery might make it more difficult to plan optimal eye movements between sequential words in a line of text. Thus, the relationship between crowding and text-spacing might reflect the benefit conferred to individuals with elevated crowding by limiting the amount of cluttered information in peripheral vision.

In summary, our results show that elevated crowding is one mechanism that contributes to reading difficulties and is the main predictor of whether a subject will benefit from increased text-spacing. Our results further imply that it is important to develop effective methods to characterize the collection of impairments that contribute to an individual's reading difficulties. By doing so, we can design personalized interventions that specifically target these mechanisms akin to personalized medicine, rather than searching for a single, one-size-fits-all approach to dyslexia treatment.

\section{Acknowledgements}

This work was supported by research grants from Microsoft Advanced Reading Technologies to JDY. We would like to thank Altaire Anderson for help with data collection, Scott Murray, Kevin Larson and Emily Kubota for helpful discussion on the manuscript, Geoffrey Boynton and John Palmer for critical input on the design of the cueing paradigm, and the University of Washington Disability Resources for Students (DRS) and Dyslexic Advantage for help with subject recruitment.

\section{Author Contributions}


All authors developed the study concept and contributed to the study design. Data collection was performed by DJS, and SJJ and ALW performed the data analysis and interpretation under the supervision of JDY. SJJ, ALW, and JDY wrote the manuscript. All authors approved the final version of the manuscript for submission.

\section{References}

Amitay, S., Ben-Yehudah, G., Banai, K., \& Ahissar, M. (2002). Disabled readers suffer from visual and auditory impairments but not from a specific magnocellular deficit. Brain: $A$ Journal of Neurology, 125(Pt 10), 2272-2285.

Bosse, M.-L., Tainturier, M. J., \& Valdois, S. (2007). Developmental dyslexia: the visual attention span deficit hypothesis. Cognition, 104(2), 198-230. doi:10.1016/j.cognition.2006.05.009

Bouma, H. (1970). Interaction effects in parafoveal letter recognition. Nature, 226(5241), 177178.

Bouma, H., \& Legein, C. P. (1977). Foveal and parafoveal recognition of letters and words by dyslexics and by average readers. Neuropsychologia, 15(1), 69-80.

Brainard, D. H. (1997). The Psychophysics Toolbox. Spatial Vision, 10(4), 433-436.

Demb, J. B., Boynton, G. M., \& Heeger, D. J. (1997). Brain activity in visual cortex predicts individual differences in reading performance. Proceedings of the National Academy of Sciences of the United States of America, 94(24), 13363-13366.

Doron, A., Manassi, M., Herzog, M. H., \& Ahissar, M. (2015). Intact crowding and temporal masking in dyslexia. Journal of Vision, 15(14), 13. doi:10.1167/15.14.13

Draper, N. R., \& Smith, H. (1998). Applied regression analysis. Hoboken, NJ: Wiley-Interscience.

Eden, G. F., VanMeter, J. W., Rumsey, J. M., Maisog, J. M., Woods, R. P., \& Zeffiro, T. A. (1996). Abnormal processing of visual motion in dyslexia revealed by functional brain imaging. Nature, 382(6586), 66-69. doi:10.1038/382066a0

Franceschini, S., Gori, S., Ruffino, M., Pedrolli, K., \& Facoetti, A. (2012). A causal link between visual spatial attention and reading acquisition. Current Biology, 22(9), 814-819. doi:10.1016/j.cub.2012.03.013

García-Pérez, M. A. (1998). Forced-choice staircases with fixed step sizes: asymptotic and small-sample properties. Vision Research, 38(12), 1861-1881.

Gori, S., \& Facoetti, A. (2015). How the visual aspects can be crucial in reading acquisition? The intriguing case of crowding and developmental dyslexia. Journal of Vision, 15(1), 15.1.8. doi:10.1167/15.1.8

Grainger, J., Dufau, S., \& Ziegler, J. C. (2016). A Vision of Reading. Trends in Cognitive Sciences, 20(3), 171-179. doi:10.1016/j.tics.2015.12.008

Herzog, M. H., Sayim, B., Manassi, M., \& Chicherov, V. (2016). What crowds in crowding? Journal of Vision, 16(11), 25. doi:10.1167/16.11.25

Joo, S. J., Donnelly, P. M., \& Yeatman, J. D. (2017). The causal relationship between dyslexia and motion perception reconsidered. Scientific Reports, 7(1), 4185. doi:10.1038/s41598- 
017-04471-5

Legge, G. E., Mansfield, J. S., \& Chung, S. T. (2001). Psychophysics of reading. XX. Linking letter recognition to reading speed in central and peripheral vision. Vision Research, 41(6), 725-743.

Levi, D. M. (2008). Crowding--an essential bottleneck for object recognition: a mini-review. Vision Research, 48(5), 635-654. doi:10.1016/j.visres.2007.12.009

Lovegrove, W. J., Bowling, A., Badcock, D., \& Blackwood, M. (1980). Specific reading disability: differences in contrast sensitivity as a function of spatial frequency. Science, 210(4468), 439-440.

Martelli, M., Di Filippo, G., Spinelli, D., \& Zoccolotti, P. (2009). Crowding, reading, and developmental dyslexia. Journal of Vision, 9(4), 14.1-18. doi:10.1167/9.4.14

Olulade, O. A., Napoliello, E. M., \& Eden, G. F. (2013). Abnormal visual motion processing is not a cause of dyslexia. Neuron, 79(1), 180-190. doi:10.1016/j.neuron.2013.05.002

Paulesu, E., Démonet, J. F., Fazio, F., McCrory, E., Chanoine, V., Brunswick, N., ... Frith, U. (2001). Dyslexia: cultural diversity and biological unity. Science, 291(5511), 2165-2167. doi:10.1126/science. 1057179

Pelli, D. G. (1997). The VideoToolbox software for visual psychophysics: transforming numbers into movies. Spatial Vision, 10(4), 437-442. doi:10.1163/156856897X00366

Pelli, D. G., \& Tillman, K. A. (2008). The uncrowded window of object recognition. Nature Neuroscience, 11(10), 1129-1135. doi:10.1038/nn.2187

Pennington, B. F. (2006). From single to multiple deficit models of developmental disorders. Cognition, 101(2), 385-413. doi:10.1016/j.cognition.2006.04.008

Pennington, B. F., \& Bishop, D. V. M. (2009). Relations among speech, language, and reading disorders. Annual Review of Psychology, 60, 283-306. doi:10.1146/annurev.psych.60.110707.163548

Perea, M., \& Gomez, P. (2012a). Increasing interletter spacing facilitates encoding of words. Psychonomic Bulletin \& Review, 19(2), 332-338. doi:10.3758/s13423-011-0214-6

Perea, M., \& Gomez, P. (2012b). Subtle increases in interletter spacing facilitate the encoding of words during normal reading. Plos One, 7(10), e47568. doi:10.1371/journal.pone.0047568

Peterson, R. L., \& Pennington, B. F. (2012). Developmental dyslexia. The Lancet, 379(9830), 1997-2007. doi:10.1016/S0140-6736(12)60198-6

Ramus, F., Rosen, S., Dakin, S. C., Day, B. L., Castellote, J. M., White, S., \& Frith, U. (2003). Theories of developmental dyslexia: insights from a multiple case study of dyslexic adults. Brain: A Journal of Neurology, 126(Pt 4), 841-865. doi:10.1093/brain/awg076

Roach, N. W., \& Hogben, J. H. (2004). Attentional modulation of visual processing in adult dyslexia: a spatial-cuing deficit. Psychological Science, 15(10), 650-654. doi:10.1111/j.0956-7976.2004.00735.x

Siok, W. T., Perfetti, C. A., Jin, Z., \& Tan, L. H. (2004). Biological abnormality of impaired reading is constrained by culture. Nature, 431(7004), 71-76. doi:10.1038/nature02865

Skottun, B. C. (2000). The magnocellular deficit theory of dyslexia: the evidence from contrast sensitivity. Vision Research, 40(1), 111-127.

Spinelli, D., De Luca, M., Judica, A., \& Zoccolotti, P. (2002). Crowding effects on word identification in developmental dyslexia. Cortex, 38(2), 179-200. 
Stein, J., \& Walsh, V. (1997). To see but not to read; the magnocellular theory of dyslexia. Trends in Neurosciences, 20(4), 147-152. doi:10.1016/S0166-2236(96)01005-3

Vellutino, F. R., Fletcher, J. M., Snowling, M. J., \& Scanlon, D. M. (2004). Specific reading disability (dyslexia): what have we learned in the past four decades? Journal of Child Psychology and Psychiatry, and Allied Disciplines, 45(1), 2-40.

Vidyasagar, T. R., \& Pammer, K. (2010). Dyslexia: a deficit in visuo-spatial attention, not in phonological processing. Trends in Cognitive Sciences, 14(2), 57-63. doi:10.1016/j.tics.2009.12.003

Wandell, B. A., \& Le, R. K. (2017). Diagnosing the neural circuitry of reading. Neuron, 96(2), 298-311. doi:10.1016/j.neuron.2017.08.007

Watson, A. B., \& Pelli, D. G. (1983). QUEST: a Bayesian adaptive psychometric method. Perception \& Psychophysics, 33(2), 113-120. doi:10.3758/BF03202828

Whitney, D., \& Levi, D. M. (2011). Visual crowding: a fundamental limit on conscious perception and object recognition. Trends in Cognitive Sciences, 15(4), 160-168. doi:10.1016/j.tics.2011.02.005

Yu, D., Cheung, S.-H., Legge, G. E., \& Chung, S. T. L. (2007). Effect of letter spacing on visual span and reading speed. Journal of Vision, 7(2), 2.1-10. doi:10.1167/7.2.2

Zorzi, M., Barbiero, C., Facoetti, A., Lonciari, I., Carrozzi, M., Montico, M., ... Ziegler, J. C. (2012). Extra-large letter spacing improves reading in dyslexia. Proceedings of the National Academy of Sciences of the United States of America, 109(28), 11455-11459. doi:10.1073/pnas.1205566109 


\section{Figure legends}

Figure 1. Crowding is correlated with reading skills. (A) Crowding stimuli. After 500 ms of fixation on the central mark, crowding stimuli appeared at either the left or the right side of the display. The stimuli disappeared after $150 \mathrm{~ms}$ and subjects reported the direction of the gap (up or down) using a joystick. We measured critical spacing at two target eccentricities $\left(6^{\circ}\right.$ or $\left.10^{\circ}\right)$ in separate blocks of trials. (B) Critical spacing increases with target eccentricity (means: 1.82 at $6^{\circ} ; 4.55$ at $10^{\circ}$ ). The error bars represent bootstrapped $68 \%$ confidence intervals (Cls). (C-D) The relationship between reading skills and critical spacing in adults. The y-axis indicates the TOWRE composite score, a standardized index of reading speed and accuracy that is normed by age $($ population mean $=100, S D=15)$. The $x$-axis represents critical spacing at $6^{\circ}(C)$ and $10^{\circ}$ (D) eccentricity. Gray circles show individual data points, and the black line is the best-fitting linear regression line to the data. (E) The relationship between reading skills and critical spacing in children. The $x$-axis indicates critical spacing at $6^{\circ}$ eccentricity.

Figure 2. Increased text-spacing improves reading performance. (A-D) Examples of real word $(A, B)$ and pseudoword $(C, D)$ lists with normal $(A, C)$ and increased $(B, D)$ text-spacing. $(E)$ Accuracy for each list. Gray and black bars represent proportion correct for real words and pseudowords, respectively. Filled and empty bars indicate increased and normal text-spacing, respectively. Accuracy was not affected by text-spacing. Error bars are bootstrapped $68 \%$ Cis, representing the between subject variance (equivalent to \pm 1 SEM). (F) Reading time for each list. Increased text-spacing lowered reading time for pseudowords within individuals. Error bars are bootstrapped $68 \%$ Cls. ${ }^{*} p<0.05$ based on a paired sample t-test.

Figure 3. Increased text-spacing improves reading speed for poor readers with elevated crowding. (A) Subjects with low reading skills, but not typical readers, benefit from increased 
text-spacing. The $y$-axis is the difference between reading time for normal and increased textspacing. Red and blue bars are for poor readers and typical readers, respectively. Poor readers: TOWRE index standard score < 95; Typical readers: TOWRE > 95. (B) Reading time for pseudowords with normal (x-axis) and increased (y-axis) text-spacing. Orange points represent individuals with high critical spacing and green points represent individuals with low critical spacing, based on a median split. The shaded region is a bootstrapped $95 \%$ confidence interval for the best-fitting line. The dashed line is the unity line. For slow readers there is a substantial benefit of increased text -spacing, demonstrated by the significant deviation of the linear fit from the identity line. (C) Critical spacing predicts the effect of text-spacing. The black line is the bestfitting regression. (D) Text-spacing benefits are pronounced for subjects with high critical spacing and not present in the low critical spacing group. Error bars are bootstrapped $68 \% \mathrm{Cls}$.

Figure 4. Text-spacing effects are not due to impaired spatial attention. (A) The stimuli and procedure for the cueing experiment. The search array $(83 \mathrm{~ms})$ comprised 7 vertically oriented distractor Gabors and a tilted target Gabor. Observers reported the direction of the target's tilt. In the cued condition, a $100 \%$ valid peripheral pre-cue (red dot) appeared in the $33 \mathrm{~ms}$ preceding the search array. In the uncued condition there was no pre-cue. Orientation thresholds were measured with a staircase procedure (See Methods). (B) Orientation thresholds in the cued and uncued conditions. The dashed line represents the unity line. All subjects' thresholds were lower in the cued condition, suggesting that our subjects effectively used the cue to focus spatial attention and exclude distractors. (C) The attention index (cueing effect) does not predict text-spacing effects. (D) The attention index does not predict critical spacing. 